\title{
Alternative conceptual design of a magnet support structure for plasma fusion devices of stellarator type
}

\author{
Nikola Jaksic*, Boris Mendelevitch, Jörg Tretter \\ Max-Planck-Institute for Plasma Physics, EURATOM Association, Boltzmannstraße 2, D-85748 Garching, Germany
}

\section{A R T I C L E I N F O}

Article history:

Available online 2 March 2011

\section{Keywords:}

Conceptual design

Magnet support structure

Stellarator

Plasma fusion

\begin{abstract}
A B S T R A C T
Engineering design of magnet coil support structures for plasma fusion devices of the stellarator type are at present an important task in stellarator hardware R\&D activities. In particular this is one of the basic core components in developing the stellarator's line in view of a robust and reliable fusion reactor.

Based on long time experience in design and structural analyses of stellarator magnet systems and their support structure, the authors are proposing in this paper an alternative conceptual design for the magnet support structure. This paper describes the basic assumptions that a conceptual design of a magnet support structure has to fulfil. In this context, essential experiences gathered during manufacturing and assembly of the magnet support structure for a current stellarator fusion device engineered at Max Planck Institute for Plasma Physics are taken into account. The concept provides flexibility in matters of readjustment and positional optimization of the magnet coils during the assembly phase and potentially during the operation. The flexibility during the assembly phase allows a simplification of technical requirements and performance criteria which may result in a reduction of costs and improved reliability of a stellarator based power device.
\end{abstract}

(C) 2011 Published by Elsevier B.V.

\section{Introduction}

Worldwide plasma fusion research is presently focused on two different types of experimental devices - tokamak and stellarator and their different principles of plasma confinement. In a tokamak, magnetic fields are generated by external coils and the intrinsic current, which circulates through the plasma and heats it at the same time. In a stellarator, the confining magnetic fields are generated by exclusively external coils without any or relatively small circulating current in the plasma. The system is thus heated by external sources only.

Stellarator devices operate in a steady-state mode in contrast to the tokamak family working in a pulsed mode. The operating modes are probably the most important difference between both principles, in particular with regard to fusion reactors which need a real steady-state operating mode.

Notwithstanding the advantages in operation mode, the fact that stellarators rely entirely on magnetic fields produced by external coils, requires a more complex shape for the coils than in tokamaks. The complex coil shapes and the necessary support structures are at present a challenging task in R\&D of stellarator devices in comparison with tokamaks.

\footnotetext{
* Corresponding author. Tel.: +49 8932991134; fax: +49 8932991620.

E-mail address: nikola.jaksic@ipp.mpg.de (N. Jaksic).
}

This paper introduces a proposal for an alternative conceptual design for the magnet support structure based on long experience in the design and structural analyses of stellarator magnet systems $[1,2]$. This conceptual design has been developed particularly with regard to a modular quasi-helical symmetric stellarator reactor, based on the physics optimization of stellarators published in $[3,4]$.

Nevertheless, this conceptual design is in general universally applicable and it is independent from the device modularity and size.

\section{Conceptual design description}

At the beginning of the considerations, some basic assumptions should be determined.

First, it is assumed that the device (fusion reactor power plant) consists of only one single modular field coil system. Presently, most of the stellarator reactor studies favour one single modular coil system, e.g. according to [5] a helias stellarator fusion reactor would contain solely 50 modular field coils.

Secondly, it is assumed in consideration of steady-state operation that the device is superconducting and operates at cryogenic temperature.

Thirdly, each superconducting coil winding pack has an individually designed coil housing, made of e.g. stainless steel, which is sufficiently stiff regarding the allowable deformations. These modular coil housings are fully separated from each other and have to 


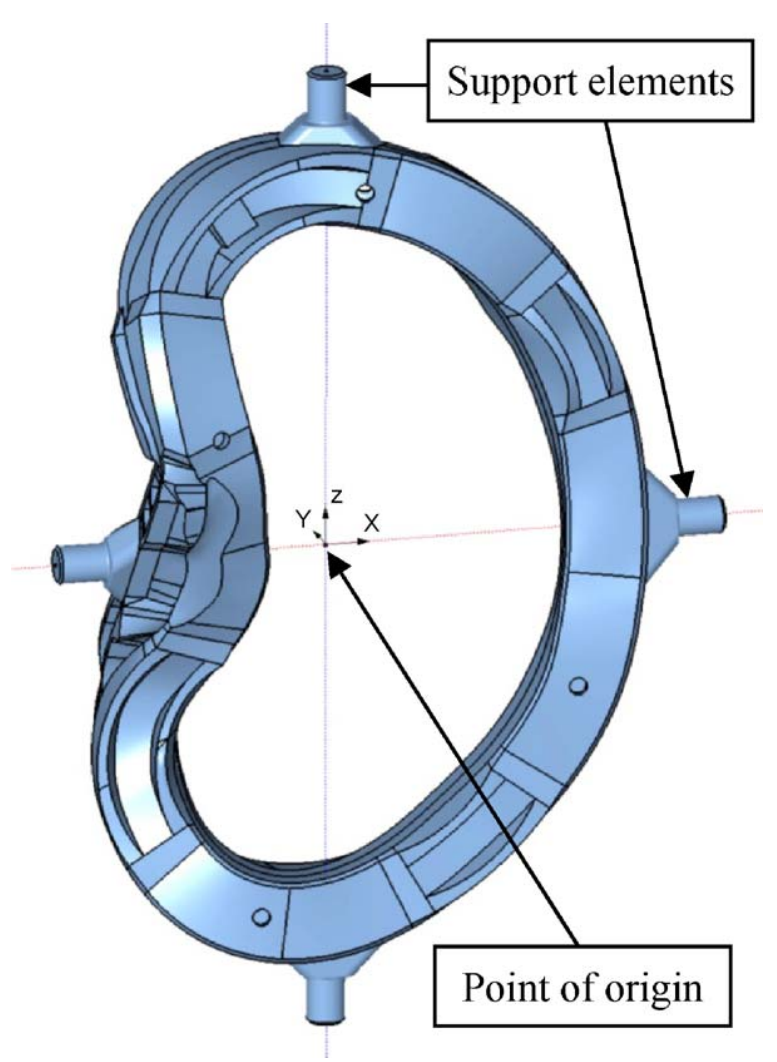

Fig. 1. Coil housing type 1 with support elements.

carry the primary loads caused by electromagnetic forces due to the current of the coils within the magnetic field. An immediate interaction of the coil housings within the alternative coil support concept is not allowed. This fact defines the minimum distance between two adjacent coil winding packs. This value can be determined first and foremost when the coil system is determinate. In general for a coil system described under [6] the minimum distance should amount to approx. $100 \mathrm{~mm}$ and for a correspondingly scaled reactor coil system [5] approx. $350 \mathrm{~mm}$.

Fourthly, the general conditions used in the present considerations imply, that the whole coil arrangement shapes a torus or a quasi-torus and the grand total of electromagnetic forces of the coil arrangement is equal to zero.

Fifth, the embedding of the coil winding pack into the coil housing is not an issue of consideration in this paper. It is assumed that a procedure similar to the one described in [7] is used for this purpose.

The basic principle of the alternative conceptual design of the coil magnet support structure is displayed in Fig. 1.

The coil housing geometry shown in Fig. 1 is an existing CAD model which provides a basis for conceptual design explanations. The original geometry has been retrofitted by support elements used for the alternative support concept. The position of the support elements at the coil housing circumference is determined, e.g. for coil system described in [6] as follows:

The horizontal and vertical couple of corresponding support elements have a common axis. The point of intersection of both axes lies exactly at the magnet field axis. This point in Fig. 1 is the origin of local coordinate system and these axes are coincident with local coordinate axis $x$ and local coordinate axis $z$.

The only decisive guideline for the definition of the origin and orientation of the axis for coil housing support elements is the impact of the coil deformation in operating state on the electromagnetic field degradation. The parameters of the local coordinate

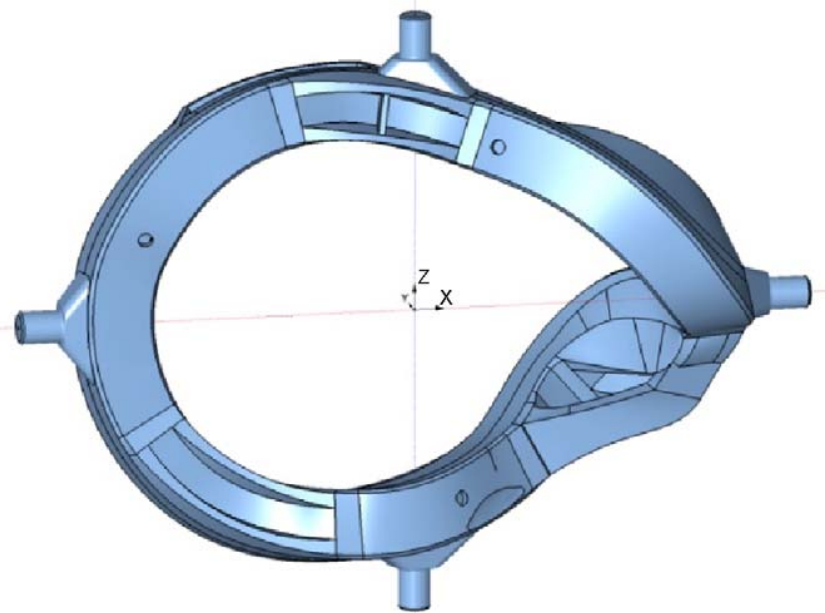

Fig. 2. Coil hosing type 5 with support elements.

system for coil support elements have to be found in an iterative procedure where the minimum for magnetic field degradation is searched in a function (Eq. (1)) with the origin and the local axes orientation as unknown variables.

$\operatorname{MFD}=\min \{f$ (orig., orient. $)\}$

where MFD is the magnetic field degradation, orig. is the origin and orient. is the orientation of the local coordinate system.

In Eq. (1) it is assumed that the basic stiffness of a coil housing is largely predefined, although a solution of Eq. (1) may require a correction of the coil housing stiffness, i.e. some iteration solving steps have to be performed until the aim is achieved.

The orientation of the local coordinate axes has to fulfil some additional conditions. First, these axes have to penetrate the coil housing body 4 times and secondly, these 4 places at the coil housing surface have to be suitable for placement of the support elements like in Figs. 1 and 2.

A first approach in searching for the appropriate origin and orientation of the local coordinate system for the definition of the coil support elements provide the principle axes of inertia and the centre of gravity of the coil winding pack. It can be shown that this method provides a reasonable first approach. It is in the nature of things that the centre of gravity of a coil is positioned close to the magnetic field axis and the principle axes of inertia will penetrate almost always the coil winding pack body. The only remaining task is to find the appropriate position on the coil housing for placement of the support elements. For the under [6] described and here shown coil system, it was almost always possible to find a suitable place at the coil housing surface by slight correction of the axes of orientation while maintaining the coil centre of gravity as the origin of the local coordinate system. Fig. 2 shows the position of the support elements for the coil type 5 of the above mentioned coil system.

In addition to the individually designed and sufficiently stiff coil housing an appropriate structure is necessary. This structure should allow for a precise positioning of the central pathway of each of the individually shaped coils, in order to fulfil the basic demand - the precise localization of the magnetic field confining the plasma.

A structural concept with properties mentioned above is shown in Fig. 3.

Such a shape of lateral stiffeners is produced necessarily from linking each support element shown in Fig. 1, proceeding from one adjacent coil housing to the other. The radial stiffeners facilitate the structure's spatial stiffness and add up to a cage-like structure. 


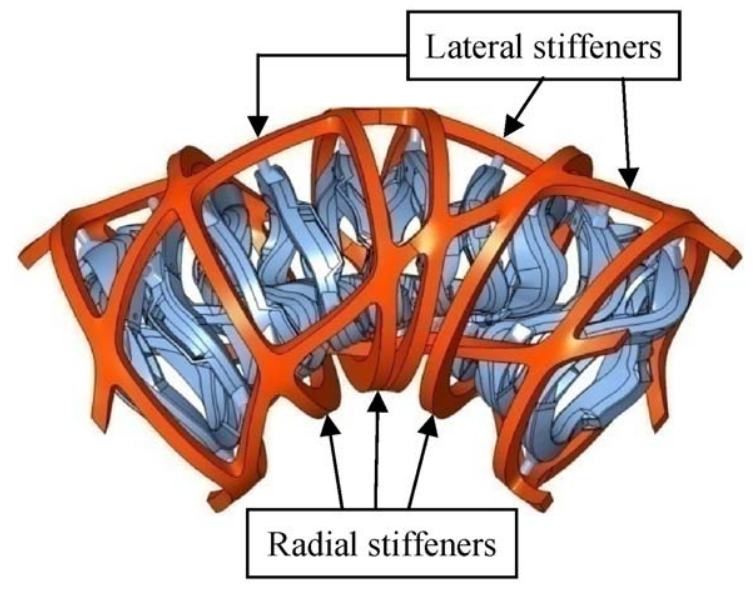

Fig. 3. CAD-model of the master coil support structure - 1 module (top view).

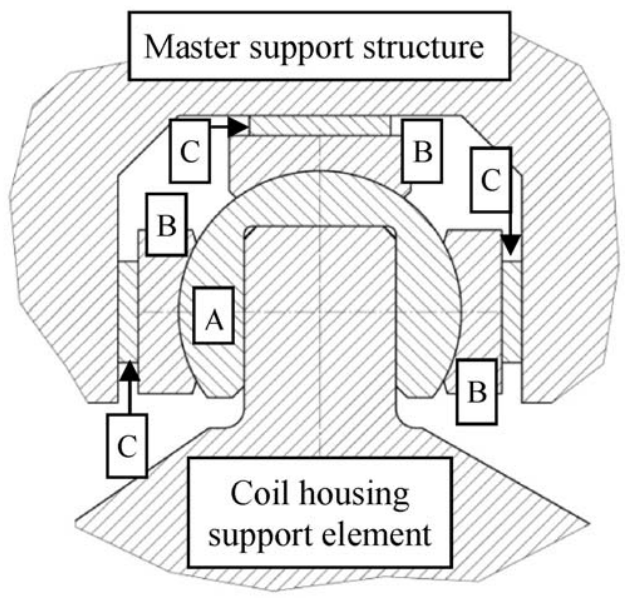

Fig. 4. Joining element.

There are 4 lateral stiffeners at the coils circumference and 6 radial stiffeners for each module.

The embedding of each single coil housing into the master support structure is realized using a special designed joining element shown in Fig. 4, as a principle draft.

These elements have all 6 degrees of freedom during assembly of the coil housings into the master support structure, and provide moment free support of the coil housings during operation.

In principle, the joining element is made up of three parts: core A is a sphere with a hole, the counterparts B and the spacers C. The spacers have a variable thickness whose final dimension is determined during the assembly phase.

In particular the design of the master coil support has to provide sufficient stiffness as well as comfortable access to the interior of the device - for plasma diagnostics in case of an experimental device or for maintenance in case of a fusion reactor. E.g. in the current design the radial stiffeners are positioned radially outward, predominantly behind the contour of the coil housings.

\section{Conceptual design validation}

The finite element model (FE-model) based on the design presented in Fig. 3 has been prepared (Fig. 5).

The main features of this superconducting coil system [6] are summarized in Table 1.

The FE-model is reduced to $1 / 10$ th of the whole coil system by the introduction of special boundary conditions. This is possible

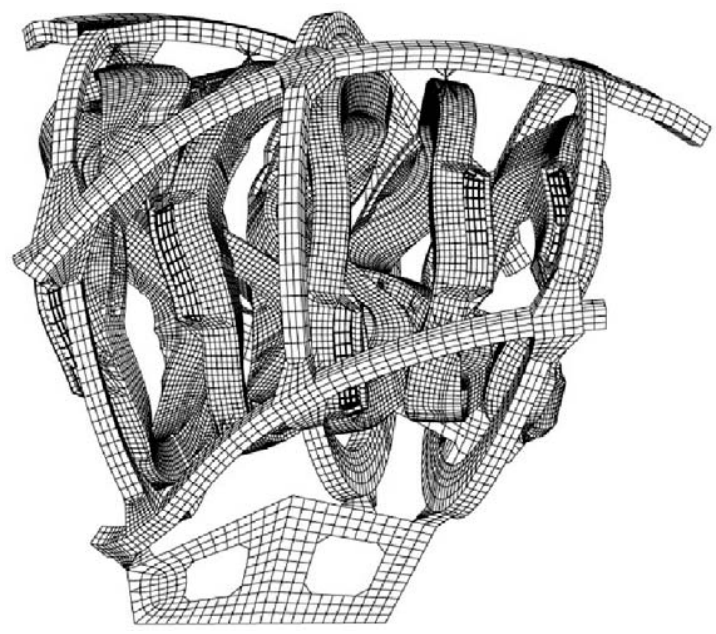

Fig. 5. FE-model - 1/2 module (top view).

because the original geometry and the loading imply specific symmetry conditions described in [8]. Due to validation, the master coil support structure is also designed according to the same symmetry conditions. The FE-model of the coil winding pack and coil housings chosen for present validation is modelled with all significant details [1] needed for such an analysis. The lateral stiffeners in the FEmodel have a constant rectangular cross-section with side lengths of $160 \mathrm{~mm}$. The radial stiffeners have the same cross-section except the back side of the middle and right stiffener in Fig. 5. The crosssection changes its height from, at the top $160 \mathrm{~mm}$, over the middle plane at $320 \mathrm{~mm}$ back to $160 \mathrm{~mm}$ at the bottom.

A few optimization steps have shown that a plate-like rib at the middle plane of the device with the effective thickness of $300 \mathrm{~mm}$ and an approx. width of $700 \mathrm{~mm}$ substantially stabilizes the whole master coil support structure. The junction elements have been simply modelled by beam finite elements. The moment free support of the coil housings was only taken partially into account in this first validation step.

A stress-strain analysis of the proposed structure has been performed to prove, in principle, the validity of the concept. The displacement plot in Fig. 6 gives first information about the behaviour of the structure. The maximal displacement in the structure adds up to $12.6 \mathrm{~mm}$. These quite small displacements of a structure, only roughly adapted for this analysis, demonstrate the potential of the proposed magnet support concept - e.g. the displacements of the first coil housing (from right) are below $6.0 \mathrm{~mm}$, although this coil housing is only moderately optimized in terms of the current conceptual design.

The stresses within the structure shown in Fig. 7 (von Mises stress) prove the high potential for optimization of the structure as well. The stresses are far below the allowable limits of $900 \mathrm{MPa}$ for e.g. a cryogenic steel 1.4429 . The local stress peaks of up to $2000 \mathrm{MPa}$ are caused by the simplification of the joining structural parts by means of beam finite elements.

Table 1

Modular coil system parameters.

Number of modular field coils

Number of field periods

Mean major torus radius

Main radius of module field coils

Max. current in the conductor

Max. magnetic induction on axis

Max. magnetic induction at the coil

Max. force on a coil

50

5

$5.5 \mathrm{~m}$

$1.5 \mathrm{~m}$

$18.2 \mathrm{kA}$

$3.0 \mathrm{~T}$

$6.7 \mathrm{~T}$

$3.76 \mathrm{MN}$ 


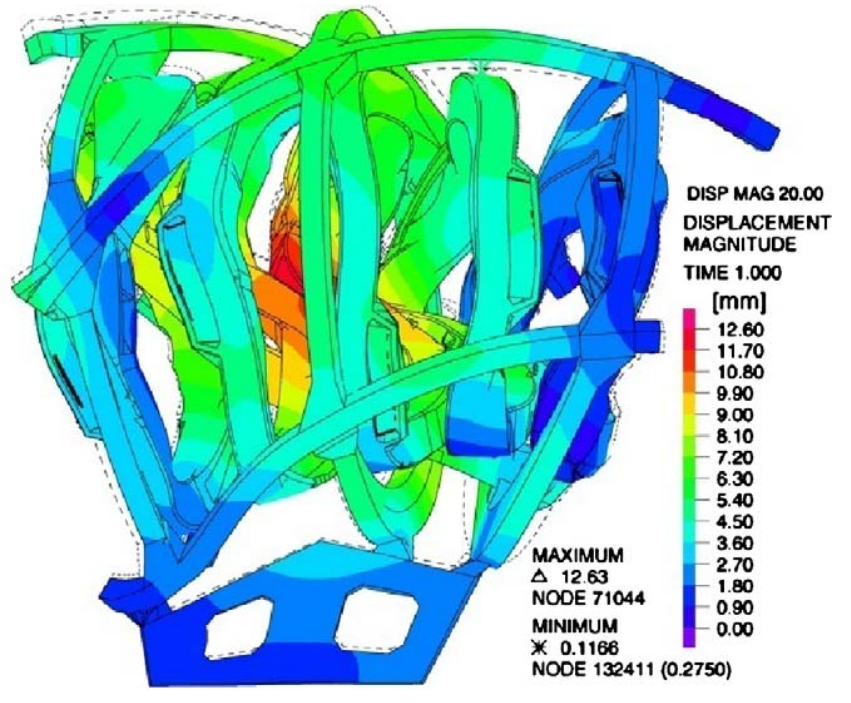

Fig. 6. The displacements of the structure.

\section{Conceptual design discussion}

The main feature of the proposed coil support structure conceptual design is its high flexibility in consideration of the assembly and adjustment phase. This conceptual design ensures precise positioning of the central pathway of each of the individually shaped coil housings. Manufacturing tolerances following from positional accuracy requirements can be shifted to a more economical range. All positional inaccuracies of the central coil pathway can be compensated by the support concept, except for the shape tolerances of the coil winding pack itself.

Probably the most important condition in the development of the present conceptual design was to provide a design which can be manufactured with conventional industrial tolerances, methods and tools.

Furthermore, this master support concept is designed so, that all main loads in the structure are compressive forces. This fact renders the design essentially easier than in case of tractive loads, because it facilitates the adoption of the form locking principle during assembly. I.e. there are no additional connecting elements like bolts or weld seems in the assembly needed. The loads acting in

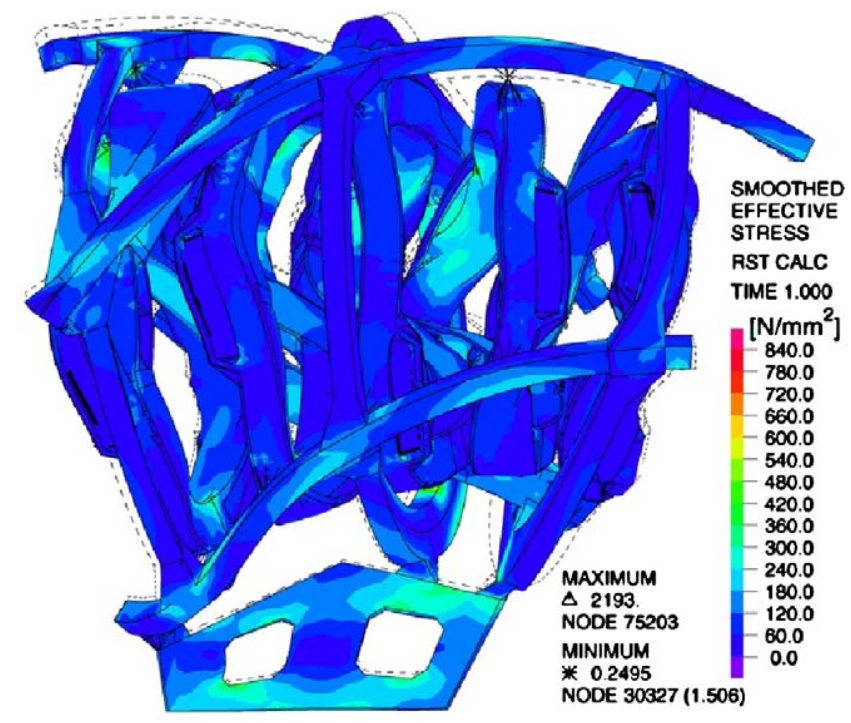

Fig. 7. Von Mises stress distribution in the structure. the structure are transferred simply by surface contact of two adjacent structural elements. Especially if the order of magnitude of the forces in the structure is equal or higher than $100 \mathrm{MN}$, the form locking principle is particularly advantageously.

Moreover, the development, the optimization and the analysis of the individual structural parts becomes considerably easier - e.g. each single coil housing could be optimized individually, outside of the remaining structure. Only the optimization of the master support structure requires all structural parts.

The validation analysis in terms of displacements shows, that this support system can be applied even to so-called compact devices. Because of this fact, the support of two adjacent coil housings can be realized also in regions with small coil housing distances and hence uncontrolled contact can be avoided.

The master coil support concept presented in this paper with its 4 lateral stiffeners is an example. The number of the lateral and radial stiffeners has to be carefully determined in the respective application on a specific magnet coil system. E.g. a reduction of the lateral stiffeners from 4 to 3 and an additional reduction of the radial stiffeners can considerably simplify the whole support design and increase the accessibility to the interior of the device.

The joining element is the key part of the support system and the design in Fig. 4 presents the basic functionality of this structural part only. The hole in the sphere (A) is not necessary from a functional point of view. The reason for the introduction of this hole is the simplification of the manufacturing and the assembly processes. Moreover, in a particular case, it is possible, to optimize the coil housing so, that the moments at the coil housing support elements are negligible - the sphere elements become superfluous. Generally, the substitution of the sphere element with a simpler element for a specific application retaining all here described functionalities is conceivable.

The here presented coil support conceptual design becomes more attractive, if the outer cryostat remains within the master support structure. In this case, readjustment of the central coil pathway is feasible during operation, without the interruption of the coils cooling, merely the coils should be deenergised.

\section{Conclusions}

The conceptual design of the magnet support structure for plasma fusion devices of the stellarator type presented in this paper should contribute to the general efforts in the development of the stellarator line. Despite the complex coil geometries, stellarators remain attractive for realization of devices up to reactor size.

The intention of the development was to design a structure which entirely obeys the guidelines known under the keyword "feasibility"; not only in terms of basic principles but also in terms of conventional industrial methods of manufacturing. The support design concept provides an assembly-friendly structure which guarantees precise positioning of the central coil pathway even in case of uncertainties during manufacture.

The basic principles, design methods and conditions which such a magnet support structure has to fulfil have been described. In addition, a validation analysis on the basis of an existing coil system has been exemplified. This conceptual design validation demonstrates the optimization potential of the proposed structural concept.

Generally, this conceptual design is universally applicable and independent from the device modularity and size.

\section{References}

[1] N. Jaksic, et al., Design analysis of the support structure stressed by large superconducting coils for a plasma fusion experiment, Computer \& Structures 81 (2003) 697-714. 
[2] N. Jaksic, et al., Nonlinear global-local finite element analysis of a future plasma fusion experiment, Computer \& Structures 72 (1999) 209-231.

[3] J. Nührenberg, et al., Overview on Wendelstein 7-X theory, Transactions of Fusion Technology 27 (1995) 71-78.

[4] G. Grieger, et al., Physics optimisation stellarators, Physics of Fluids B4 (1992) 2081-2091.

[5] G. Grieger, et al., HELIAS stellarator reactor studies and related European technology studies, Fusion Engineering and Design 25 (1994) 73-84.
[6] C. Beidler, et al., Physics and engineering design for Wendelstein7-X, Fusion Technology 17 (1989) 140-168.

[7] L. Wegener, et al., Final design and construction of the Wendelstein7-X coils, Fusion Engineering and Design 58-59 (2001) 225-230.

[8] N. Jaksic, Definition der Randbedingungen bei einer FE-Strukturanalyse durch Nutzung der Symmetriebedingungen einer Stellaratoranordnung, Interne Publikation, IPPZ/3, February 1997. 